\title{
BIG BANG NUCLEOSYNTHESIS AND LEPTON NUMBER ASYMMETRY IN THE UNIVERSE
}

\author{
K. KOHRI， M. KAWASAKI \\ Institute for Cosmic Ray Research, University of Tokyo, Tanashi, \\ Tokyo 188, Japan \\ AND \\ KATSUHIKO SATO \\ Department of Physics and Research Center for the Early Universe, \\ School of Science, University of Tokyo, Tokyo 113, Japan
}

Recently it has been reported that there may be a discrepancy between big bang nucleosynthesis theory and observations (BBN crisis) (Hata et al., 1995). One way to solve the discrepancy might be to adopt some modifications of standard physics used in SBBN (Kawasaki et al., 1997). We show that BBN predictions agree with the primordial abundances of light elements, ${ }^{4} \mathrm{He}, \mathrm{D},{ }^{3} \mathrm{He}$ and ${ }^{7} \mathrm{Li}$ inferred from the observational data if the electron neutrino has a net chemical potential $\xi_{\nu_{e}}$ due to lepton asymmetry (Kohri et al., 1997). We study BBN with the effects of the neutrino degeneracy in details using Monte Carlo simulation and make a likelihood analysis using the most recent data. We estimate that $\xi_{\nu_{e}}=0.043_{-0.040}^{+0.040}(95 \%$ C.L.) and $\Omega_{b} h^{2}=0.015_{-0.003}^{+0.006}$ (95\% C.L.) adopting the presolar Deuterium abundance as the primordial values. If we adopted the low $\mathrm{D}$ abundance which is obtained by the observation of the high redshift QSO absorption systems, $\xi_{\nu_{e}}=0.060_{-0.042}^{+0.034}(95 \%$ C.L. $)$ and $\Omega_{b} h^{2}=0.020_{-0.004}^{+0.006}$. The estimated chemical potential of $\nu_{e}$ is about $10^{-5} \mathrm{eV}$ which is much smaller than experiments can detect $(\sim 1 \mathrm{eV})$. In other words, BBN gives the most stringent constraint on the chemical potential of $\nu_{e}$.

\section{References}

Hata, N., Scherrer, R. J., Steigman, G., Thomas, D., Walker, T. P., Bludman, S. \& Langacker, P. 1995, Phys. Rev. Lett. 75, 3977.

Kohri, K., Kawasaki, M. \& Sato, K. 1997, ApJ. in press, astro-ph/9612237.

Kawasaki, M., Kohri, K., \& Sato, K. 1997, astro-ph/9705148. 